OPEN ACCESS

Edited by:

Claudia Fabiani,

University of Siena, Italy

Reviewed by:

Katarzyna Krysik,

Wojewódzki Szpital Specjalistyczny nr

5 Sosnowiec, Poland Beatrice Gallo,

NHS Foundation Trust,

United Kingdom

*Correspondence:

Alessandro Arrigo

alessandro.arrigo@hotmail.com

Specialty section: This article was submitted to Ophthalmology,

a section of the journal

Frontiers in Medicine

Received: 19 June 2021

Accepted: 30 July 2021

Published: 19 August 2021

Citation:

Arrigo A, Aragona E, Capone L, Di Biase C, Lattanzio R and Bandello $F$ (2021) Intraocular Pressure Changes Are Predictive of Ocular Hypertension Onset After Fluocinolone Acetonide Implant: Significant Cutoffs and the Role of Previous DEX Implant. Front. Med. 8:725349. doi: 10.3389/fmed.2021.725349

\section{Intraocular Pressure Changes Are Predictive of Ocular Hypertension Onset After Fluocinolone Acetonide Implant: Significant Cutoffs and the Role of Previous DEX Implant}

\author{
Alessandro Arrigo *, Emanuela Aragona, Luigi Capone, Carlo Di Biase, \\ Rosangela Lattanzio and Francesco Bandello \\ Department of Ophthalmology, IRCCS San Raffaele Scientific Institute, Vita-Salute San Raffaele University, Milan, Italy
}

Background: Fluocinolone acetonide (FAC) implant represents a long-term strategy for the management of diabetic macular edema (DME). Because of the 3-year duration, the careful monitoring of the intraocular pressure (IOP) is necessary. The main aim of the study was to provide quantitative IOP cutoffs associated with the onset of IOP increases.

Methods: The study was retrospectively conducted with 2-year of follow-up. We separately considered eyes with good IOP control (Group 1), eyes requiring IOP-lowering medications (Group 2) and eyes undergoing IOP-lowering surgery (Group 3). The statistical analysis assessed Delta\% IOP changes over the 2-year follow-up. ROC analysis was performed to detect significant cutoffs associated with Group 2 and Group 3. IOP changes occurring after a previously administered dexamethasone (DEX) implant were also evaluated.

Results: We included 48 eyes (48 patients), stratified as follows: Group 1 (25/48; 52\%), Group 2 (19/48; 40\%) and Group 3 (4/48; 8\%). ROC analysis performed on IOP values detected 2-months later DEX implant showed a mean Delta IOP increase $>24 \%$ significantly associated with IOP-lowering medications after FAc implant, whereas a mean Delta IOP increase $>35 \%$ was significantly associated with IOP-lowering surgery after FAc implant. With respect to IOP changes occurred after FAc implant, our $\mathrm{ROC}$ analysis showed a mean Delta IOP increase $>8 \%$ significantly associated with IOP-lowering medications, whereas a mean Delta IOP increase $>15 \%$ was significantly associated with IOP-lowering surgery. DEX-related IOP changes showed $52 \%$ sensitivity and $100 \%$ specificity of FAc-related IOP increases.

Conclusions: IOP changes provides clinically relevant cutoffs associated with the onset of FAc-related IOP increases.

Keywords: diabetic macular edema, fluocinolone acetonide implant, intraocular pressure, IOP lowering medications, trabeculectomy, dexamethasone implant 


\section{INTRODUCTION}

Diabetic macular edema (DME) is a common complication of diabetic retinopathy (DR), currently managed by means of intravitreal anti-VEGF injections and corticosteroids (1-3). The more recently introduced fluocinolone acetonide (FAc) $0.19 \mathrm{mg}$ intravitreal drug-delivery system (ILUVIEN ${ }^{\circledR}$; Alimera Sciences, Inc., Alpharetta, GA, USA) provided a step forward regarding the long-term management of DME, guaranteeing up to 3 years of treatment duration (4-9). Although efficacy and safety have been largely demonstrated (4-9), the main issue regarding the employment of FAc implant is the management of intraocular pressure (IOP) and the prediction of IOP-related complications. Estimating the overall incidence of IOP elevation requiring lowering topical medications, the current data reported at least $20-30 \%$ of treated eyes (8-12). Furthermore, the overall incidence of uncontrolled IOP requiring IOP-lowering surgery is reported at least in the $2-5 \%$ of cases $(8-12)$. If these numbers appear relatively small, thus making ophthalmologists confident with FAc treatment, if applied to larger number of eyes, the incidence of IOP elevations and IOP-lowering surgeries might turn out to be characterized by absolute high number of patients. Indeed, although small, IOP-related complications are remarkably higher in FAc-treated DME eyes, compared with dexamethasone (DEX) implant. It is undoubted that the IOP-related complications are more frequent in FAc-treated eyes, compared with DEX implants; taking, for example, a recent study collecting data from a high number of DEX-treated eyes, the cumulative probability of IOP increases $\geq 21, \geq 25$ and $\geq 35 \mathrm{mmHg}$ was found equal to 50 $60 \%, 25-30 \%$, and $6-7 \%$ at $12-24$ months, respectively, with an overall incidence of IOP-lowering surgery of $0.9 \%$ (13). In this scenario, IOP changes registered after DEX implants were found useful to try to predict IOP behavior following FAc implant (814), although few data are available regarding the real predictivity of DEX implant.

This study aimed to analyze in deep a selected cohort of DME eyes treated by FAc implant and reaching 2 years of follow-up, in order to (I) analyze IOP changes and reporting IOP-related complications and management; (II) establish quantitative IOP cutoffs suggestive of IOP-related events onset occurring during the follow-up; (III) establish the role of previous DEX implant in terms of IOP cutoffs predictive of complications onset; (IV) assess the predictivity of DEX-related IOP elevation on IOP increases after FAc implant.

\section{MATERIALS AND METHODS}

The study was designed as retrospective, cohort study. DME patients treated with FAc implant at the Department of Ophthalmology, IRCCS San Raffaele Scientific Institute, Milan, Italy, were included into the study. The study was approved by the local ethical committee (MIRD2020) and was conducted in accordance with Helsinki declaration. All the patients signed an informed consent before the inclusion into the study.

The inclusion criteria were eyes treated by FAc implant, followed for at least 2-years, with mandatory complete followup visits conducted every 2 months, with a positive history of at least one DEX implant administered before FAc implant. All the eyes were pseudophakic, accordingly with the Italian guidelines for the use of FAc implant. Exclusion criteria were the presence of media opacities, any kind of ocular surgery occurred during the 2 years of FAc follow-up, with the only exception of selective laser trabeculoplasty (SLT) or IOP-lowering surgery, any ophthalmic or systemic disease potentially affecting the results of the study (ocular hypertension, uveitis, other maculopathies or retinopathies over than DR, uncontrolled diabetes mellitus, uncontrolled systemic arterial hypertension, other endocrine system disorders). All the patients underwent complete ophthalmologic examination including ETDRS best corrected visual acuity (BCVA), slit lamp evaluation, Goldmann applanation tonometry, structural OCT (Spectralis HRA, Heidelberg Engineering; Heidelberg, Germany) with radial, raster and dense scans with high number of frames (ART > 25) and enhanced depth imaging (EDI) to highlight choroidal structures. Structural OCT images were used to extract central macular thickness (CMT) values. IOP was measured at least twice by expert ophthalmologists. During the follow-up visits, the definition of ocular hypertension was established on the IOP value $>20 \mathrm{mmHg}$. IOP was always measured in the morning for all the eyes. The criterion to start IOP lowering medication was the detection of ocular hypertension at follow-up visits. The choice of the starting drug was performed accordingly with ophthalmologists' discretion. All the eyes started with only one IOP lowering medication; combination therapy was adopted when registering poor response to monotherapy (still presence of ocular hypertension). Eyes were switched to IOP lowering surgery (trabeculectomy) for those eyes who have failed maximal medical therapy, confirmed by clinical and instrumental evaluations. Age, gender, systemic hypertension, type and duration of diabetes mellitus (DM), glycate hemoglobin (HbA1c), DR stage, previous vitrectomy, previous panretinal photocoagulation (PRP), and previous macular grid laser were considered as fixed factors. All the statistical analyses were performed by means of SPSS software package (SPSS, Illinois, USA). From the consecutive measures of IOP, we also calculated the delta values, obtained subtracting the measurements performed during the follow-up visits from the baseline values. These were used to assess the percentage changes of IOP over the 2-year follow-up and to perform the cutoff analysis. Continuous variables were assessed by unpaired $T$ test. ANOVA test was used to separately analyze three subgroups, including eyes not requiring IOP-lowering medications, eyes requiring IOP-lowering medications and eyes underwent IOPlowering surgery (trabeculectomy). Bonferroni correction was applied to assess multiple comparisons. The relationship among the considered variables was explored by Tau-Kendall correlation test. Moreover, we perform a ROC analysis to detect statistically significant IOP cutoffs, based on delta IOP values, associated with the need of IOP-lowering medications or the need of IOPlowering surgery. ROC analysis was performed both considering IOP values registered after FAc implant and IOP values measured before and 2 months after the previous DEX implant. In case of multiple DEX implants, we considered the IOP measured after the first DEX implant. The predictive value of DEX implant 
was tested reporting the true positive and the false positive cases of DEX-related IOP increases followed by FAc-related IOP increases. Statistical significance was set at $p<0.05$.

\section{RESULTS}

One-hundred and thirteen eyes of 79 DME patients were considered. Forty-two out of 113 eyes (37\%) required IOPlowering medications, whereas $12 / 113$ eyes (11\%) underwent IOP-lowering surgery.

Among all these patients, 48 eyes of 48 patients (25 males; mean age $68 \pm 8$ years) met the inclusion criteria and were considered for the analyses. The clinical data are extensively reported in Table 1. Considering that the mean IOP significantly increased during the first year of follow-up since FAc implant $(p<0.01), 23$ out of 48 eyes $(48 \%)$ required IOP-lowering medications, whereas $4 / 48$ eyes ( $8 \%)$ underwent IOP-lowering surgery. The mean time from the first IOP-lowering medication was $10 \pm 8$ months, whereas the mean time from IOP-lowering surgery was $12 \pm 2$ months. The cohort of DME eyes experienced statistically significant improvements both of LogMAR BCVA ( $p$ $=0.02)$ and CMT $(p<0.01)$ during the first year of follow-up, maintained during the second year of follow-up (both $p>0.05$ ).

We stratified the cohort of eyes as follows: Group 1 (not requiring IOP-lowering medications; 25/48 eyes; 52\%), Group 2 (requiring IOP-lowering medications; 19/48 eyes; 40\%) and Group 3 (requiring IOP-lowering surgery; $4 / 48$ eyes; $8 \%$ ). As reported in Table 2, clinical features, previous history, Baseline LogMAR BCVA and CMT values were similar among the three groups (all $p>0.05$ ). Group 1 and Group 2 experienced statistically significant BCVA improvement (both $p<0.05$ ) during the first year of follow-up, maintaining similar values during the second year (both $p>0.05$ ). On the other side, Group 3 showed stable BCVA values both during the first and the second year of follow-up (both $p>0.05$ ). All the three Groups underwent significant CMT improvement during the first year of follow-up (all $p<0.01$ ), keeping CMT stable at 2-year follow-up (all $p>0.05$ ).

Looking at the previous IOP changes detected 2 months later the first DEX implant (Table 3), Group 1 showed stable IOP values $(p>0.05)$, with a mean Delta IOP of $4 \%$; Group 2 and Group 3 showed statistically significant IOP increases (both $p<0.01$ ), with Delta IOP values of 42 and 59\%, respectively. Group 3 overall showed the highest IOP increase after DEX implant $(p<0.01)$. In all the cases, the IOP came back to normal values, thus not requiring IOP-lowering medications after the four months of DEX implant.

The three Groups started with similar IOP values before FAc implant (all $p>0.05)$. Statistically significant IOP increases were registered already at the 2-month follow-up in Group 2 and Group 3 (both $p<0.01$ ). Group 1 maintained IOP values within the normal range for the entire 2-year follow-up, reaching the highest but not significant mean Delta IOP values of $12 \%$ at month $20(p>0.05)$. With respect to Group 2 and Group 3, although the absolute IOP value was significantly different from month 10 (17 vs. $21 \mathrm{mmHg} ; p<0.01$ ), mean Delta IOP was
TABLE 1 | Clinical and Imaging data.

\begin{tabular}{|c|c|c|}
\hline Parameter & Mean \pm STD & $\begin{array}{c}P \text {-value (baseline } \\
\text { vs. } 24 \mathrm{~m} \text { ) }\end{array}$ \\
\hline Age (years) & $68 \pm 8$ & \\
\hline Gender (M/F) & $25 / 23$ & \\
\hline IOP Increase (\%) & 23/48 (48\%) & \\
\hline Trabeculectomy (\%) & 4/48 (8\%) & \\
\hline DM Type (1/2) & 19/29 (40\%/60\%) & \\
\hline $\begin{array}{l}\text { Duration of DM } \\
\text { (months) }\end{array}$ & $\begin{array}{c}25 \pm 14 \text { (36 } \pm 9 \text { for } \\
\text { DMT1 and } 18 \pm 10 \text { for } \\
\text { DMT2) }\end{array}$ & \\
\hline Arterial hypertension & 30/48 (63\%) & \\
\hline $\begin{array}{l}\text { Panretinal } \\
\text { photocoagulation }\end{array}$ & 28/48 (58\%) & \\
\hline $\begin{array}{l}\text { Focal grid macular } \\
\text { laser }\end{array}$ & $24 / 48(50 \%)$ & \\
\hline Vitrectomy & 8/48 (17\%) & \\
\hline DR type (NPDR/PDR) & 29/19 (60\%/40\%) & \\
\hline $\mathrm{HbA1c}(\%)$ & $7.1 \pm 0.9$ & \\
\hline baseline_IOP (mmHg) & $14 \pm 2$ & $p<0.01$ \\
\hline 12m_IOP (mmHg) & $17 \pm 4$ & \\
\hline 24m_IOP (mmHg) & $16 \pm 4$ & \\
\hline $\begin{array}{l}\text { baseline_LogMAR } \\
\text { BCVA }\end{array}$ & $0.55 \pm 0.39$ & $p=0.02$ \\
\hline 12m_LogMAR BCVA & $0.48 \pm 0.37$ & \\
\hline 24m_LogMAR BCVA & $0.47 \pm 0.36$ & \\
\hline baseline_CMT ( $\mu \mathrm{m})$ & $529 \pm 212$ & $p<0.01$ \\
\hline 12m_CMT $(\mu \mathrm{m})$ & $338 \pm 114$ & \\
\hline 24m_CMT $(\mu \mathrm{m})$ & $323 \pm 130$ & \\
\hline
\end{tabular}

IOP, Intraocular pressure; DM, diabetes mellitus; DR, diabetic retinopathy; NPDR, nonproliferative diabetic retinopathy; $P D R$, proliferative diabetic retinopathy; $B C V A$, bestcorrected visual acuity; CMT, central macular thickness.

significantly different already at month 2 (11 vs. $37 \%$; $p<0.01)$. The course of IOP values is extensively reported in Table 3. At the end of the follow-up, Group 2 was characterized by absolute IOP values within the normal range $(16 \pm 3 \mathrm{mmHg}$; $p>0.05)$ and mean Delta IOP of $6 \%$, with respect to baseline. On the other side, Group 3 showed absolute values included in the upper margin of normal range $(20 \pm 4 \mathrm{mmHg}$; $\mathrm{p}>0.05)$ and maintaining remarkably higher mean Delta IOP (55\%) with respect to baseline.

We found statistically significant correlations between Delta IOP detected after DEX implant and the need both of IOPlowering medications (Tau-Kendall coeff. 0.738; $p<0.01$ ) and IOP-lowering surgery (Tau-Kendall coeff. 0.416; $p<0.01$ ), as well as between absolute IOP values after DEX implant and the need both of IOP-lowering medications (Tau-Kendall coeff. 0.658; $p<0.01$ ) and IOP-lowering surgery (Tau-Kendall coeff. $0.395 ; p<0.01$ ) (Table 4). Furthermore, both IOPlowering medications and IOP-lowering surgery significantly correlated with IOP values detected at month 2 (Tau-Kendall coeff. 0.457 and 0.334 , respectively; $p<0.01$ ) and month 4 (Tau-Kendall coeff. 0.423 and 0.384 , respectively; $p<0.01$ ). IOP-lowering medications significantly correlated also with IOP 
TABLE 2 | Clinical and imaging data of the subgroup analysis.

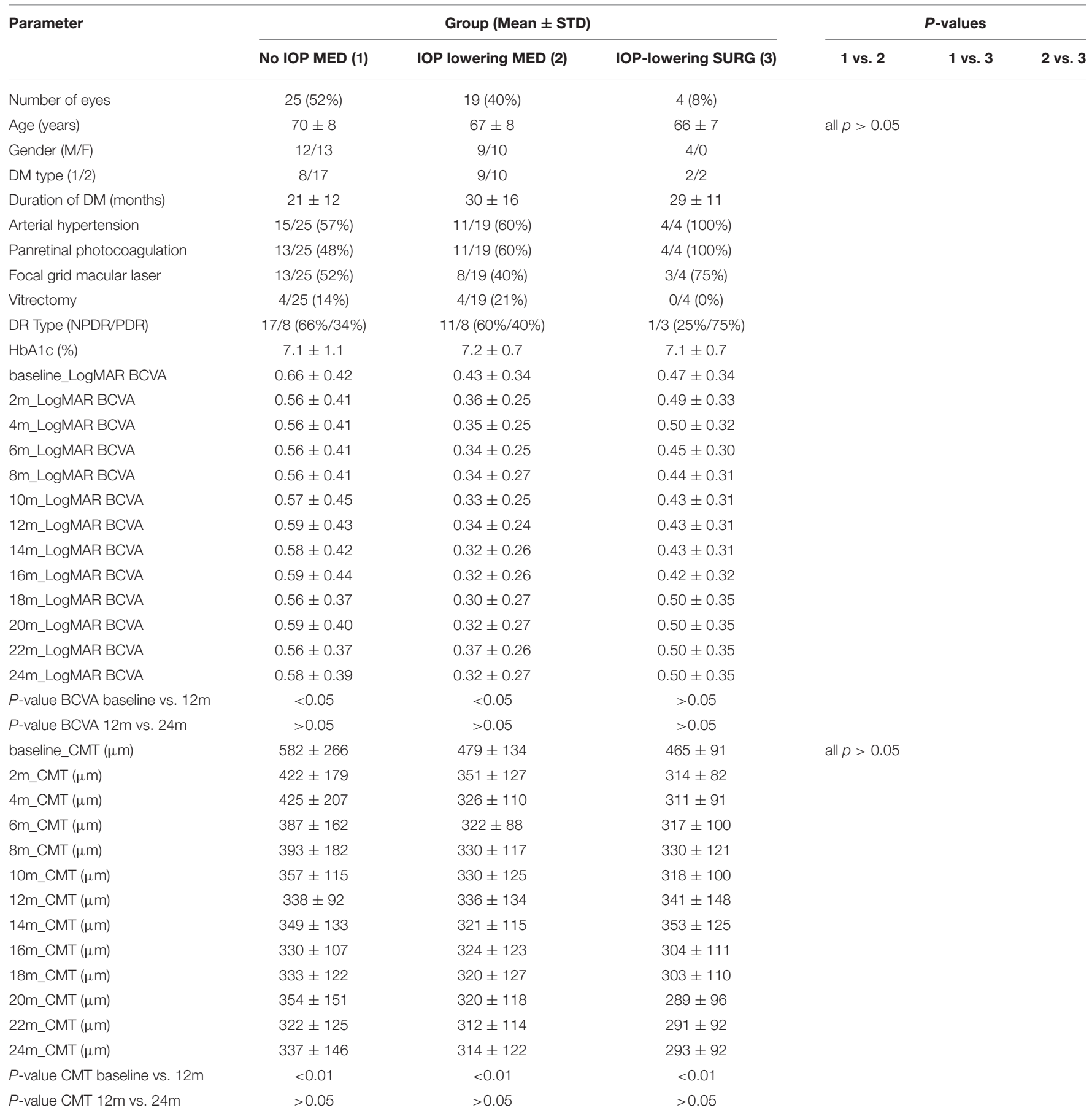

IOP, intraocular pressure; DM, diabetes mellitus; DR, diabetic retinopathy; NPDR, non-proliferative diabetic retinopathy; PDR, proliferative diabetic retinopathy; BCVA, best-corrected visual acuity; CMT, central macular thickness.

values measured at month 6 (Tau-Kendall coeff. 0.402; $p<0.01$ ), whereas IOP-lowering surgery significantly correlated with IOP values detected at month 8 (Tau-Kendall coeff. $0.327 ; p<0.01$ ) and month 10 (Tau-Kendall coeff. 0.337; $p<0.01$ ) (Table 4). No statistically significant correlations were found between IOP values and both LogMAR BCVA and CMT changes over the entire follow-up (both considering absolute values and Delta\% variations) (all $p>0.05$ ). As expected, LogMAR BCVA and CMT showed statistically significant correlations over the follow-up (cumulative Tau-Kendall coeff. 0.350; $p<0.01$ ). In addition, IOP, 
TABLE 3 | Intraocular pressure (IOP) values in the subgroup analysis.

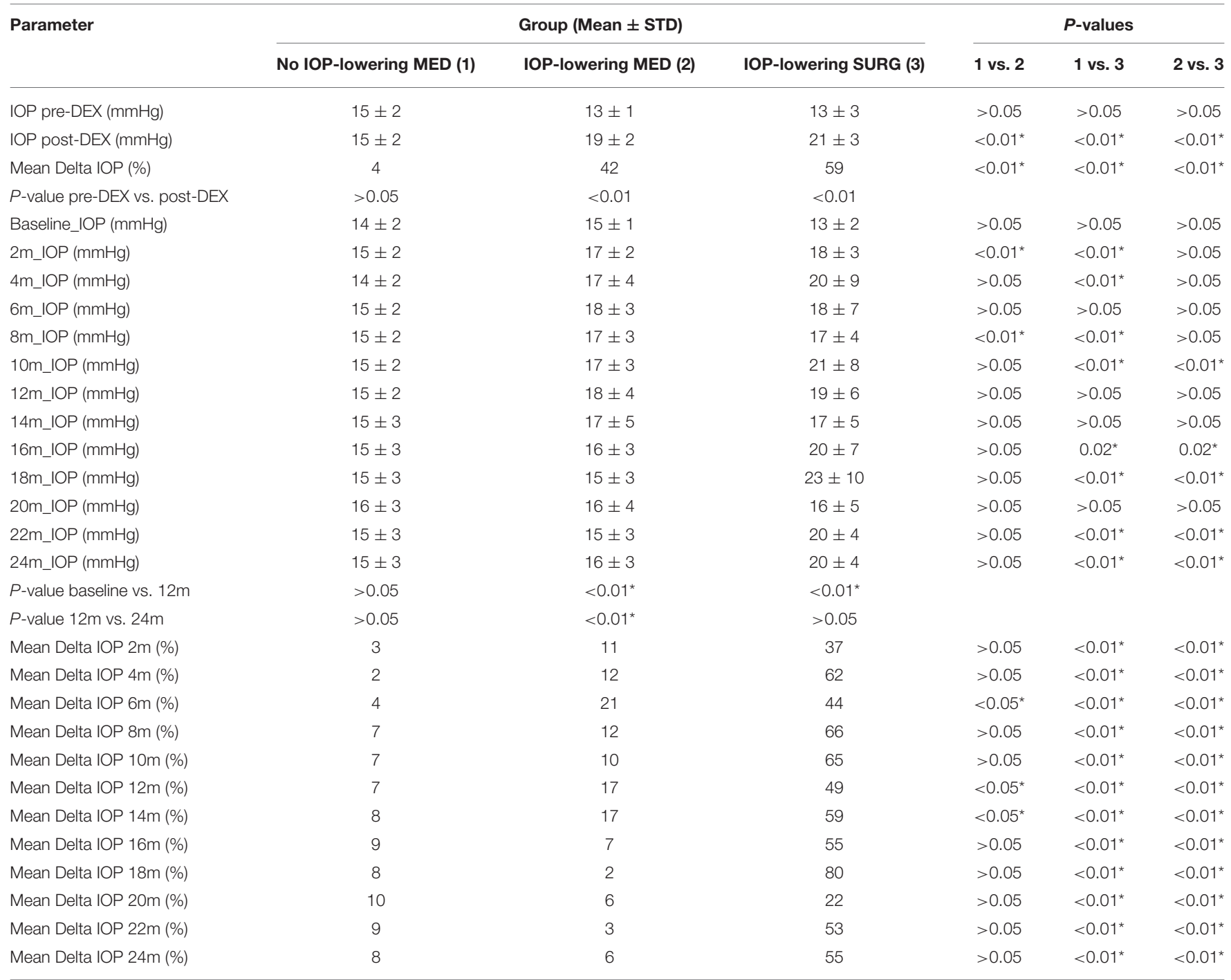

IOP, intraocular pressure; DEX, dexamethasone implant. Statistically significant values are marked by asterisks $\left(^{(*)}\right.$.

TABLE 4 | Correlation analysis.

\begin{tabular}{|c|c|c|c|c|}
\hline \multirow[t]{3}{*}{ Delta IOP\% post-DEX } & IOP-lowering MED & IOP-lowering SURG & & \\
\hline & 0.738 & 0.416 & & \\
\hline & $<0.01$ & $<0.01$ & & \\
\hline \multirow{2}{*}{ IOP post-DEX } & 0.658 & 0.395 & & \\
\hline & $<0.01$ & $<0.01$ & & \\
\hline IOP-lowering MED & $2 \mathrm{~m} \_I O P$ & $4 \mathrm{~m} \_I O P$ & $6 \mathrm{~m} \_I O P$ & \\
\hline \multirow[t]{3}{*}{ IOP-lowering SURG } & Delta\% IOP 2m & Delta\% IOP 4m & Delta\% IOP 8m & Delta\% IOP 10m \\
\hline & 0.334 & 0.384 & 0.327 & 0.337 \\
\hline & $<0.01$ & $<0.01$ & $<0.01$ & $<0.01$ \\
\hline
\end{tabular}

IOP, intraocular pressure; DEX, dexamethasone implant. 
TABLE 5 | ROC analysis detecting statistically significant IOP cutoffs associated with the need of IOP-lowering medications or IOP-lowering surgery after FAc implant.

\begin{tabular}{|c|c|c|c|c|c|}
\hline Cutoff & & ROC value (\%) & Sensitivity & Specificity & $P$-value \\
\hline \multirow{2}{*}{$\begin{array}{l}\text { Delta IOP\% post-DEX } \\
\text { associated with IOP lowering } \\
\text { medications after FAc implant }\end{array}$} & & 24.04 & 0.95 & 0.94 & $<0.01$ \\
\hline & & 26.79 & 0.91 & 0.95 & \\
\hline \multirow{2}{*}{$\begin{array}{l}\text { Delta IOP\% post-DEX } \\
\text { associated with IOP-lowering } \\
\text { surgery after FAc implant }\end{array}$} & & 30.95 & 0.96 & 0.67 & $<0.01$ \\
\hline & & 34.52 & 0.83 & 0.73 & \\
\hline \multirow{5}{*}{$\begin{array}{l}\text { Delta IOP\% after FAc implant } \\
\text { associated with IOP lowering } \\
\text { medications }\end{array}$} & & 7.5 & 0.67 & 0.71 & \\
\hline & & 10.41 & 0.67 & 0.76 & \\
\hline & Delta\% IOP 6m & 6.9 & 0.71 & 0.67 & $<0.01$ \\
\hline & & 9.82 & 0.71 & 0.71 & \\
\hline & & 12.92 & 0.67 & 0.71 & \\
\hline \multirow{8}{*}{$\begin{array}{l}\text { Delta IOP\% after FAc implant } \\
\text { associated with IOP-lowering } \\
\text { surgery }\end{array}$} & & 12.92 & 0.83 & 0.70 & \\
\hline & & 14.36 & 0.67 & 0.75 & \\
\hline & Delta\% IOP 8m & 15.48 & 0.83 & 0.73 & $<0.01$ \\
\hline & & 17.71 & 0.83 & 0.78 & \\
\hline & & 19.38 & 0.83 & 0.81 & \\
\hline & Delta\% IOP 10m & 16.03 & 0.83 & 0.73 & $<0.01$ \\
\hline & & 17.71 & 0.83 & 0.81 & \\
\hline & & 19.38 & 0.83 & 0.87 & \\
\hline
\end{tabular}

IOP, intraocular pressure; DEX, dexamethasone implant; FAc, fluocinolone acetonide implant.

BCVA and CMT were not influenced by previous vitrectomy, previous PRP or focal laser, HbA1c values, DR or DM type, systemic health status (all $p>0.05$ ). All the considered variables were not influenced by the age $(p>0.05)$.

The results of the ROC analysis are extensively reported in Table 5. In detail, we found that a mean Delta IOP increase $>24 \%$ (sensitivity 0.95 ; specificity $0.95 ; p<0.01$ ) detected 2 months later DEX implant was significantly associated with the need of IOP-lowering medications after FAc implant. Furthermore, a mean Delta IOP increase $>35 \%$ (sensitivity 0.83 ; specificity 0.73 ; $p<0.01$ ) after 2 months from DEX implant was significantly associated with the need of IOP-lowering surgery after FAc implant. If considering the mean Delta IOP changes detected after FAc implant, 2-month ( $>8 \% ; p<0.01)$ and 6-month $(>10 \% ; p<0.01)$ values were significantly associated with the need of IOP-lowering medications. Moreover, in terms of mean Delta IOP changes detected after FAc implant associated with the need of IOP-lowering surgery, we found statistically significant cutoff values at 2 -month ( $>15 \%$; $p<0.01)$, 4 -month $(>13 \%$; $p<0.01)$, 8-month $(>18 \% ; p<0.01)$ and 10 -month $(>18 \%$; $p<0.01$ ) examinations (Table 5). ROC curves are shown in Figure 1.

In terms of DEX-related IOP increase $>20 \mathrm{mmHg}$ predictivity of FAc-related IOP increase, overall, 12/48 eyes (25\%) showed IOP increase $>20 \mathrm{mmHg} 2$ months after DEX implant. Among these eyes, $12 / 12(100 \%)$ of eyes were characterized by IOP increase $>20 \mathrm{mmHg}$ after FAc implant (9 eyes (75\%) requiring IOP-lowering medications and 3 eyes (25\%) requiring IOPlowering surgery). However, 11/48 additional eyes (23\%) were characterized by IOP values $<20 \mathrm{mmHg}$ after DEX implant, but IOP increases $>20 \mathrm{mmHg}$ after FAc implant (10 eyes (91\%) requiring IOP-lowering medications and 1 eye $(9 \%)$ requiring IOP-lowering surgery). Based on our data, DEX-related IOP changes showed the following features: sensitivity $52 \%$; specificity 100\%; Positive Predictive Value 100\%; Negative Predictive Value 69\%; Accuracy $77 \%$.

\section{DISCUSSION}

In the present study, we analyzed IOP changes occurring after FAc implant, focusing on clinically relevant cutoffs associated with the need of IOP-lowering medications or IOP-lowering surgery. Our analyses highlighted two main information. Firstly, IOP changes detected after FAc implant may be predictive of IOP control loss requiring medications or surgery. Although our ROC analysis found more timepoints associated with statistically significant IOP cutoffs, it is worth of notice that already 2-month follow-up may be clinically useful for IOP control. Indeed, a mean Delta IOP change $>8 \%$ was significantly associated with 

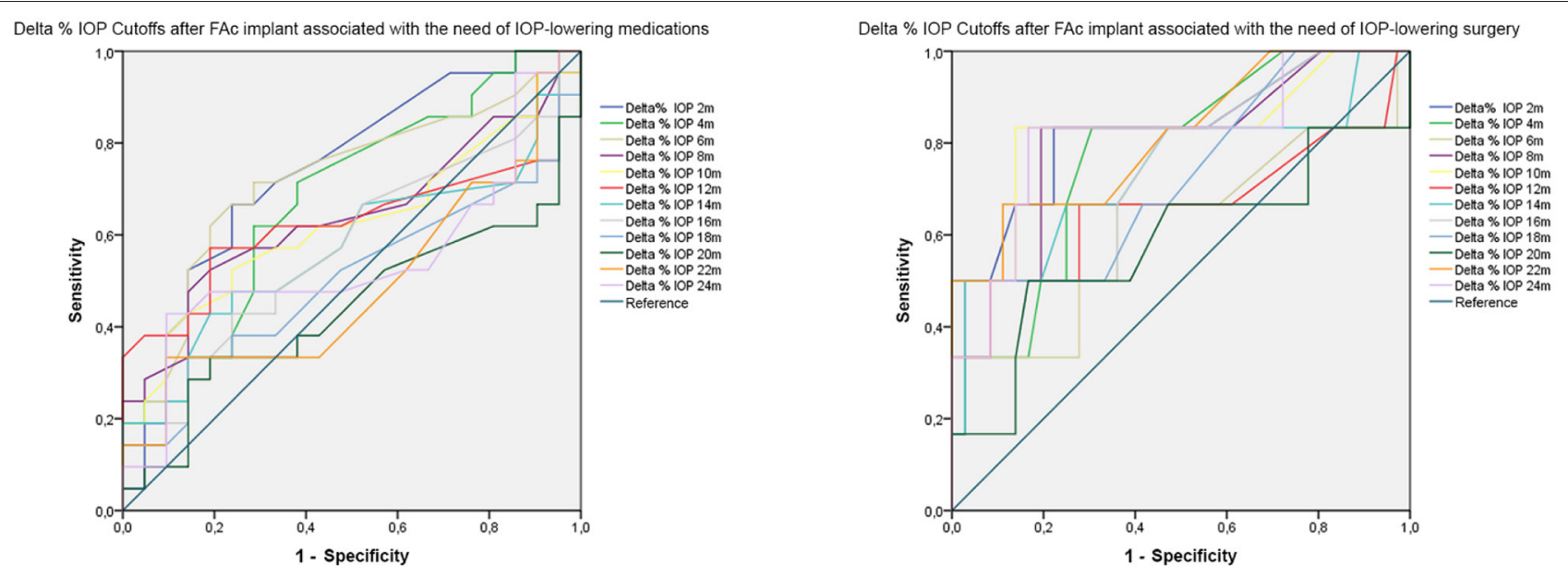

FIGURE 1 | ROC analysis of Delta\% IOP cutoffs associated with the need of IOP-lowering medications or IOP-lowering surgery after FAc implant.

the need of IOP-lowering medications, whereas a mean Delta IOP change $>15 \%$ characterized the eyes requiring IOP-lowering surgery. To the best of our knowledge, this is the first study performed considering the Delta IOP variations detected in FAcimplant setting. For this reason, the current literature is poorly useful in supporting our findings. However, our data agreed with the conclusions provided by all the previous safety assessments, which considered a careful IOP monitoring mandatory in FAc implant clinical setting (8-14).

The same methodology pointed out the effectiveness of IOP monitoring already in DEX implant setting. Hence, the second main information regarded the definition of DEX-related Delta IOP cutoffs providing useful information before FAc implant. Considering the Delta IOP changes detected 2 months later DEX implant, a mean change $>24 \%$ was significantly associated with the need of IOP-lowering medications after FAc implant, whereas a mean change $>35 \%$ was significantly associated with IOP-lowering surgery. Remarkably, considering the 2-month timepoint, the Delta IOP changes resulted higher after DEX implant than after FAc-implant. This phenomenon might be explained considering the different pharmacokinetic profiles of the two releasing systems, being at the maximal concentration after 2 months with DEX implant and resulting not yet in the plateau phase with FAc implant $(15,16)$.

Furthermore, although previous DEX implant is globally considered useful to detect cortico-responder eyes before FAc implant $(8,15)$, to the best of our knowledge, the definition of specific IOP cutoffs to assess DEX predictivity is not available in the current literature. In the present study, we reported that DEX-related IOP changes are predictive of FAc-related IOP increases with a sensitivity of $52 \%$ and a specificity of $100 \%$. This means that a IOP increase after DEX implant is a reliable warning of IOP control loss after FAc implant, but DEX implant is affected by at least $50 \%$ of false negative IOP changes. The reason of this phenomenon is unclear. Based on our correlation findings, we may exclude a possible cumulative effect of FAc molecules occurring during the follow-up. In particular, we found no correlations among IOP, BCVA and CMT behaviors, thus seeming to have no significant relationships. Furthermore, we found no linear increases of IOP values over the entire followup in all the three subgroups of eyes. In addition, the mean time of onset of clinically relevant IOP increases is relatively long (10 \pm 8 months), although showing high variability, and it is remarkably close with the mean time of loss of IOP control requiring the switching to IOP-lowering surgery (12 \pm 2 months). These observations differed from what observed and described after DEX implant, where IOP increases quickly occur and are generally well-managed by topical medications. Explaining these different IOP behaviors is quite challenging. If looking at the pharmacokinetics profiles of DEX and FAc implant, DEX is characterized by higher and not fixed release of drug (saw tooth shape) with the peak concentration occurring at the first 2 months (17). Conversely, FAc implant is characterized by a constant $0.2 \mu \mathrm{g} /$ day release, allowing to reach the steady state of concentration at month-3 (16).

Although obtained from a limited number of eyes, our data suggest that the IOP increases might be governed by other possible phenomena. Both the different pharmacokinetics and absolute concentrations of the two drugs might have an influence on the trabecular meshwork and on the regulation of aqueous production/outflow. However, we might hypothesize two different mechanisms of IOP increases. Those eyes characterized by ocular hypertension onset occurring in the first months since the FAc implant might be considered as pure cortico-responders, whereas eyes showing delayed IOP increases might be characterized by other not yet understood pathogenic sources.

The mechanisms of corticosteroid induced IOP increases are partially understood. One of the most shared hypotheses attributed the IOP elevation to a reduced aqueous outflow caused by reduced activity of the trabecular meshwork and extracellular debris accumulations (18-22). However, many other metabolic pathways have been involved in the pathogenesis of corticosteroids induced glaucoma (23), including also 
genetic predisposition (24), thus making this complication a multifactorial phenomenon.

Although the precise pathogenesis of IOP increase cannot be evinced by this study, our data strongly remarked that the reaching of IOP values $>20 \mathrm{mmHg}$ is only the last step of a more complex process already detectable after 2 months since the FAc (or DEX) implant. In this scenario, as highlighted by a recent meta-analysis, IOP monitoring is a key point in FAc setting, since the proportion of FAc-implanted eyes receiving IOP-lowering medications ranged from $7 \%$ to $46 \%$, whereas the reported need of IOP-lowering surgery ranged from $0.3 \%$ to $9.5 \%$ of cases (25). As shown by our findings, the mere detection of IOP values $>20 \mathrm{mmHg}$ is probably not sufficient to properly manage this long-term treatment. On the contrary, a definition of new IOP assessment criteria, based on the evaluation of IOP values deviation from the range considered normal for each patient, might be fundamental to plan personalized follow-up strategies and to improve FAc safety profile. For this reason, future studies should be focused on the definition of even more precise Delta IOP cutoffs, based on larger patients' cohorts.

We are aware that our study labors under several possible limitations. The first complaint may be the relatively low number of eyes and the retrospective nature of the investigation. The included eyes came from real-life settings, and underwent various treatment strategies, in accordance with ophthalmologists' discretion; these differences might exert possible influence on the clinical course of the disease after FAc implant. Furthermore, further useful information, including a deep assessment of tonometry curve, pachymetry and other more specific features of glaucoma clinical setting were not performed. In addition, we limited our observations to 2 years of follow-up, being aware about FAc therapeutic duration of 3 years. Thus, further studies should be focused on the complete assessment of IOP changes over the entire 3-year FAc follow-up.

\section{REFERENCES}

1. Das A, McGuire PG, Rangasamy S. Diabetic macular edema: pathophysiology and novel therapeutic targets. Ophthalmology. (2015) 122:1375-94. doi: 10.1016/j.ophtha.2015.03.024

2. Romero-Aroca P, Baget-Bernaldiz M, Pareja-Rios A, LopezGalvez M, Navarro-Gil R, Verges R. Diabetic macular edema pathophysiology: vasogenic versus inflammatory. J Diabetes Res. (2016) 2016:2156273. doi: 10.1155/2016/2156273

3. Schmidt-Erfurth U, Garcia-Arumi J, Bandello F, Berg K, Chakravarthy U, Gerendas BS, et al. Guidelines for the management of diabetic macular edema by the european society of retina specialists (EURETINA). Ophthalmologica. (2017) 237:185-222. doi: 10.1159/000458539

4. Campochiaro PA, Brown DM, Pearson A, Chen S, Boyer D, RuizMoreno J, et al. Sustained delivery fluocinolone acetonide vitreous inserts provide benefit for at least 3 years in patients with diabetic macular edema. Ophthalmology. (2012) 119:2125-32. doi: 10.1016/j.ophtha.2012. 04.030

5. Campochiaro PA, Brown DM, Pearson A, Ciulla T, Boyer D, Holz FG, et al. Long-term benefit of sustained-delivery fluocinolone acetonide vitreous inserts for diabetic macular edema. Ophthalmology. (2011) 118:626635.e2. doi: 10.1016/j.ophtha.2010.12.028

6. Alfaqawi F, Lip PL, Elsherbiny S, Chavan R, Mitra A, Mushtaq B. Report of 12-months efficacy and safety of intravitreal fluocinolone acetonide implant
In conclusion, our study provided quantitative IOP cutoffs resulting predicting of the need of IOP-lowering medications and IOP-lowering surgery in FAc implant setting. Patients' monitoring during the previous DEX implant was found extremely useful in terms of IOP cutoffs detection, although DEX-related IOP changes resulted highly specific but poor sensitive in predicting FAc-related IOP complications. Future studies should be conducted in order to provide new IOP monitoring criteria, thus defining new guidelines for the safe administration of FAc-implant and new strategies to prevent and to manage IOP-related complications.

\section{DATA AVAILABILITY STATEMENT}

The original contributions presented in the study are included in the article/supplementary material, further inquiries can be directed to the corresponding author/s.

\section{ETHICS STATEMENT}

The studies involving human participants were reviewed and approved by Ethical committee of IRCCS San Raffaele Scientific Institute, Milan, Italy. The patients/participants provided their written informed consent to participate in this study.

\section{AUTHOR CONTRIBUTIONS}

AA and EA: study design, data acquisition, data analysis, data interpretation, and manuscript draft. LC and CD: data analysis and data acquisition. FB and RL: data interpretation, manuscript revision, and study supervision. All authors contributed to the article and approved the submitted version.

for the treatment of chronic diabetic macular oedema: a real-world result in the United Kingdom. Eye. (2017) 31:650-6. doi: 10.1038/eye.2016.301

7. Fusi-Rubiano W, Mukherjee C, Lane M, Tsaloumas MD, Glover N, Kidess A, et al. Treating diabetic macular oedema (DMO): real world UK clinical outcomes for the $0.19 \mathrm{mg}$ fluocinolone acetonide intravitreal implant (Iluvien) at 2 years. BMC Ophthalmol. (2018) 18:62. doi: 10.1186/s12886-018-0726-1

8. Bailey C, Chakravarthy U, Lotery A, Menon G, Talks J, Medisoft Audit G. Real-world experience with $0.2 \mathrm{mug} /$ day fluocinolone acetonide intravitreal implant (ILUVIEN) in the United Kingdom. Eye. (2017) 31:170715. doi: 10.1038/eye.2017.125

9. Augustin AJ, Bopp S, Fechner M, Holz F, Sandner D, Winkgen A-M, et al. Three-year results from the Retro-IDEAL study: Realworld data from diabetic macular edema (DME) patients treated with $\operatorname{ILUVIEN}((\mathrm{R}))$ (0.19 mg fluocinolone acetonide implant). Eur J Ophthalmol. (2019) 30:38291. doi: $10.1177 / 1120672119834474$

10. Chakravarthy U, Taylor SR, Koch FHJ, Castro de Sousa JP, Bailey C; ILUVIEN Registry Safety Study (IRISS) Investigators Group. Changes in intraocular pressure after intravitreal fluocinolone acetonide (ILUVIEN): real-world experience in three European countries. Br J Ophthalmol. (2019) 103:10727. doi: 10.1136/bjophthalmol-2018-312284

11. Eaton A, Koh SS, Jimenez J, Riemann CD. The USER Study: A Chart Review of Patients Receiving a $0.2 \mu \mathrm{g} /$ day fluocinolone acetonide implant for diabetic macular edema. Ophthalmol Ther. (2019) 8:5162. doi: $10.1007 /$ s40123-018-0155-5 
12. Mansour SE, Kiernan DF, Roth DB, Eichenbaum D, Holekamp NM, Kaba $\mathrm{S}$, et al. Two-year interim safety results of the $0.2 \mu \mathrm{g} /$ day fluocinolone acetonide intravitreal implant for the treatment of diabetic macular oedema: the observational PALADIN study. Br J Ophthalmol. (2021) 105:4149. doi: 10.1136/bjophthalmol-2020-315984

13. Zarranz-Ventura J, Sala-Puigdollers A, Velazquez-Villoria D, Figueras-Roca M, Copete S, Distefano L, et al. Long-term probability of intraocular pressure elevation with the intravitreal dexamethasone implant in the real-world. PLoS ONE. (2019) 14:e0209997. doi: 10.1371/journal.pone.02 09997

14. Cicinelli MV, Rosenblatt A, Grosso D, Zollet P, Capone L, Rabiolo A, et al. The outcome of fluocinolone acetonide intravitreal implant is predicted by the response to dexamethasone implant in diabetic macular oedema. Eye. (2021). doi: 10.1038/s41433-021-01469-2. [Epub ahead of print].

15. Boyer DS, Yoon YH, Belfort R Jr, Bandello F, Maturi RK, Augustin AJ, et al. Three-year, randomized, sham-controlled trial of dexamethasone intravitreal implant in patients with diabetic macular edema. Ophthalmology. (2014) 121:1904-14. doi: 10.1016/j.ophtha.2014.04.024

16. Kane FE, Green KE. Ocular pharmacokinetics of fluocinolone acetonide following Iluvien implantation in the vitreous humor of rabbits. $J$ Ocul Pharmacol Ther. (2015) 31:11-6. doi: 10.1089/jop.2014.0100

17. Chang-Lin JE, Attar M, Acheampong AA, Robinson MR, Whitcup SM, Kuppermann BD, et al. Pharmacokinetics and pharmacodynamics of a sustained-release dexamethasone intravitreal implant. Invest Ophthalmol Vis Sci. (2011) 52:80-6. doi: 10.1167/iovs.10-5285

18. Rohen JW, Linnér E, Witmer R. Electron microscopic studies on the trabecular meshwork in two cases of corticosteroid-glaucoma. Exp Eye Res. (1973) 17:19-31. doi: 10.1016/0014-4835(73)90164-4

19. Steely HT, Browder SL, Julian MB, Miggans ST, Wilson KL, Clark AF. The effects of dexamethasone on fibronectin expression in cultured human trabecular meshwork cells. Invest Ophthalmol Vis Sci. (1992) 33:2242-50.

20. Johnson D, Gottanka J, Flügel C, Hoffmann F, Futa R, LütjenDrecoll E. Ultrastructural changes in the trabecular meshwork of human eyes treated with corticosteroids. Arch Ophthalmol. (1997) 115:375-83. doi: 10.1001/archopht.1997.01100150377011

21. Kubota T, Okabe H, Hisatomi T, Yamakiri K, Sakamoto T, Tawara A. Ultrastructure of the trabecular meshwork in secondary glaucoma eyes after intravitreal triamcinolone acetonide. J Glaucoma. (2006) 15:1179. doi: 10.1097/00061198-200604000-00007
22. Yemanyi F, Baidouri H, Burns AR, Raghunathan V. Dexamethasone and Glucocorticoid-Induced Matrix Temporally Modulate Key Integrins, Caveolins, Contractility, and Stiffness in Human Trabecular Meshwork Cells. Invest Ophthalmol Vis Sci. (2020) 61:16. doi: 10.1167/iovs.61.13.16

23. Kersey JP, Broadway DC. Corticosteroid-induced glaucoma: a review of the literature. Eye. (2006) 20:407-16. doi: 10.1038/sj.eye.6701895

24. Chan W, Wiggs JL, Sobrin L. The genetic influence on corticosteroid-induced ocular hypertension: a field positioned for discovery. Am J Ophthalmol. (2019) 202:1-5. doi: 10.1016/j.ajo.2019.02.001

25. Fallico M, Maugeri A, Lotery A, Longo A, Bonfiglio V, Russo A, et al. Fluocinolone acetonide vitreous insert for chronic diabetic macular oedema: a systematic review with meta-analysis of real-world experience. Sci Rep. (2021) 11:4800. doi: 10.1038/s41598-021-84362-y

Conflict of Interest: FB consultant for: Alcon (Fort Worth, Texas, USA), Alimera Sciences (Alpharetta, Georgia, USA), Allergan Inc (Irvine, California, USA), Farmila-Thea (Clermont-Ferrand, France), Bayer Shering-Pharma (Berlin, Germany), Bausch And Lomb (Rochester, New York, USA), Genentech (San Francisco, California, USA), Hoffmann-La-Roche (Basel, Switzerland), NovagaliPharma (Évry, France), Novartis (Basel, Switzerland), Sanofi-Aventis (Paris, France), Thrombogenics (Heverlee, Belgium), Zeiss (Dublin, USA).

The remaining authors declare that the research was conducted in the absence of any commercial or financial relationships that could be construed as a potential conflict of interest.

Publisher's Note: All claims expressed in this article are solely those of the authors and do not necessarily represent those of their affiliated organizations, or those of the publisher, the editors and the reviewers. Any product that may be evaluated in this article, or claim that may be made by its manufacturer, is not guaranteed or endorsed by the publisher.

Copyright (c) 2021 Arrigo, Aragona, Capone, Di Biase, Lattanzio and Bandello. This is an open-access article distributed under the terms of the Creative Commons Attribution License (CC BY). The use, distribution or reproduction in other forums is permitted, provided the original author(s) and the copyright owner(s) are credited and that the original publication in this journal is cited, in accordance with accepted academic practice. No use, distribution or reproduction is permitted which does not comply with these terms. 